http://jmscr.igmpublication.org/home/

ISSN (e)-2347-176x ISSN (p) 2455-0450

crossref DOI: https://dx.doi.org/10.18535/jmscr/v8i11.90

Journal Of Medical Science And Clinical Research

An Official Publication of IGM Publication

\title{
Uncommon and Unusual Pathogen - A Case of Pulmonary and Cutaneous Nocardiosis
}

\author{
Authors \\ Akhil Deshmukh ${ }^{1}$, Ravikumar Kurup ${ }^{2 *}$, Anjali Nair ${ }^{3}$, Anandhavishnu Sugathan ${ }^{4}$, \\ Aromal B Nath ${ }^{5}$, Ashwin Ramachandran ${ }^{6}$ \\ ${ }^{1,3,4,5,6}$ Department of Internal medicine, Medical College, Thiruvananthapuram, Kerala, India \\ ${ }^{2}$ Head of Department of Internal medicine, Medical College, Thiruvananthapuram, Kerala, India \\ *Corresponding author \\ Ravikumar Kurup
}

\begin{abstract}
Nocardiosis is caused by Gram-positive aerobic bacilli of the genus Nocardia, which are saprophytes living in the soil. It is a rare and deceitful disease with a localized or disseminated infection Nocardiosis is an infection affecting individuals with defective cellular immunity. The disease must be considered in the differential diagnosis in an individual presenting with skin, lung, and neurological manifestations. Here is a case of a 40year male who was on steroids for steroid-responsive encephalopathy associated with thyroperoxidase presented with fever, cough, and skin abscesses confirmed to be pulmonary and cutaneous nocardiosis. The patient improved with long-term treatment with trimethoprim-sulfamethoxazole.

Keywords: Nocardiosis; Actinomycetoma; Sinus; Abscess; antibiotics; trimethoprim; sulfamethoxazole.
\end{abstract}

\section{Introduction}

Nocardiosis is caused by Gram-positive aerobic bacilli of the genus Nocardia, which are saprophytes living in the soil. It is a rare and deceitful disease with localized or disseminated infection. Similar to anaerobic organisms of the genus Actinomyces, the Nocardia species frequently form small, but much thinner filaments than true fungi $(1-2 \mu \mathrm{m}$ vs. 3-5 $\mu \mathrm{m}$ in diameter). Nocardiosis is divided into disseminated and primary cutaneous types. Disseminated nocardiosis mainly affects immunosuppressed. HIV infection, chronic lung disease, and chronic intake of immunosuppressants are the common underlying risk factors ${ }^{(1-3)}$. Primary cutaneous nocardiosis, commonly caused by Nocardia brasiliensis, typically affects immunocompetent individuals with a history of trauma and can be subdivided into 3 clinical entities(1) lymphocutaneous infection, (2) mycetoma, and (3) superficial skin infection. Dissemination of the skin is estimated to occur in approximately $10 \%$ of patients with systemic nocardia infection, second only to CNS involvement $^{(4-7)}$.

The clinico-radiological presentation is nonspecific and is not included in the differential diagnosis because of difficulties in culturing the bacteria. Here is a case of pulmonary and cutaneous nocardiosis with satisfactory evolution 
after long term treatment with trimethoprimsulfamethoxazole.

\section{Case Description/Summary}

A 40-year male patient resident of Poovar, Kerala, and a carpenter presented fever of 3weeks duration, cough with expectoration, and breathlessness on exertion. The patient had been continuing tapering dose of prednisolone after the previous admission for steroid-responsive encephalopathy associated with thyroperoxidase (STREAT) diagnosed 1month back. There was clinical and serological evidence of this condition. Upon physical examination, there were pigmented swellings distributed over the chest and abdomen. Also had swelling of the left knee joint and a draining sinus over the back.

Chest X-ray revealed multiple nodules disseminated throughout both lungs, accompanied by blunting of right costophrenic angle. The computed tomography scan of the chest reported the presence of nodules of different sizes, some of which exhibited cavitation. Skin swellings were aspirated which demonstrated straw color thick aspirate. The right knee joint swelling was aspirated. The aspirate from the skin and joint space demonstrated gram-positive bacteria. Modified Ziehl - Neelsen staining $\left(1 \% \mathrm{H}_{2} \mathrm{SO}_{4}\right)$ showed acid-fast bacilli which are filamentous, beaded, and branching bacteria. Chocolate agar media showed the growth of chalky white colonies. MALDI-TOF was done which revealed Nocardia asteroides. Once the diagnosis of nocardiosis was made, the patient was submitted to computerised tomography of the brain, which was normal.

Thus patient was started on trimethoprimsulfamethoxazole (TMP-SMZ), ceftriaxone, and meropenem. The patient showed improvement in symptoms and TMP-SMZ was advised to continue for 6 months.

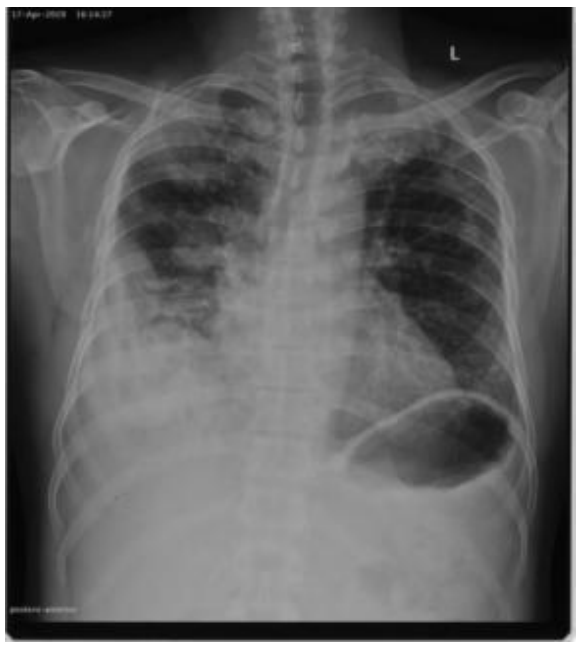

Figure 1: Chest X-ray showing multiple nodules disseminated in both lungs with opacification of right costophrenic angle.

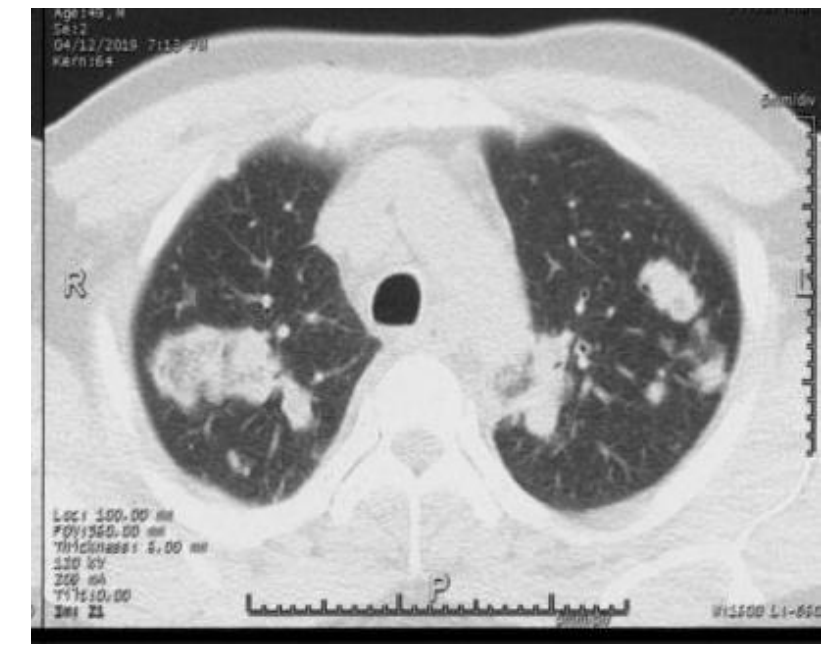

Figure 2: Computed tomography scan of the chest demonstrating diffuse nodular lesions, principally on the right side, some presenting cavitation.

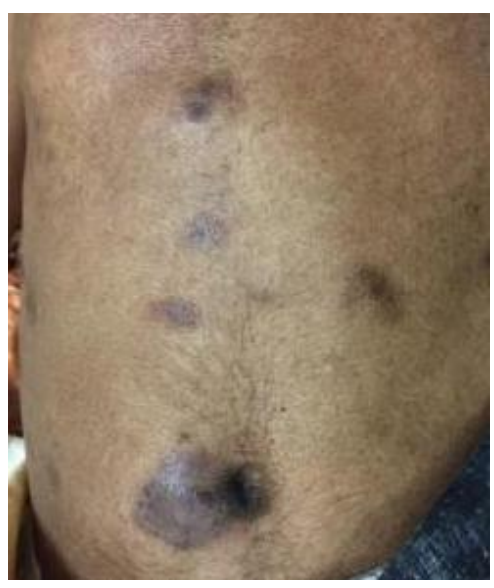

Figure 3: Skin abscess over the anterior abdominal wall. 


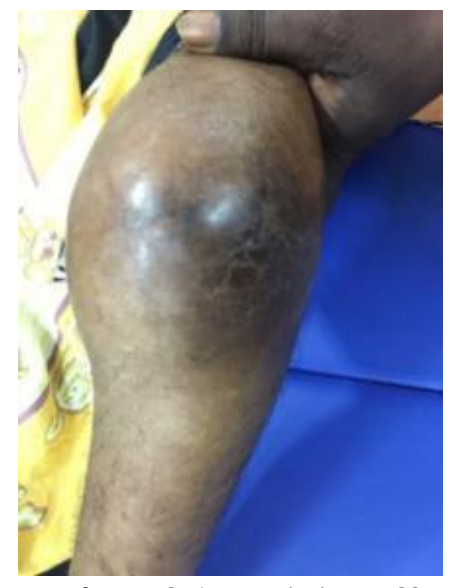

Figure 4: Left knee joint effusion.

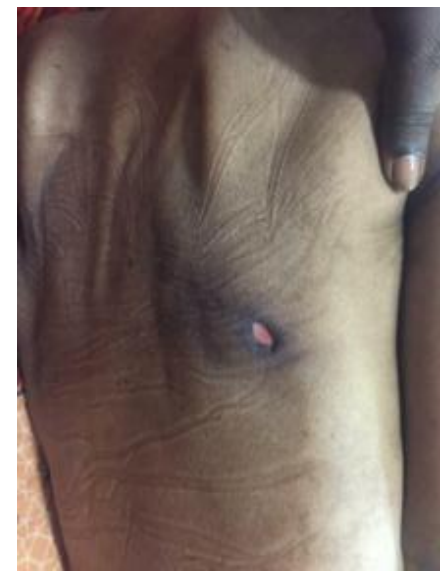

Figure 5: Sinus over the back.

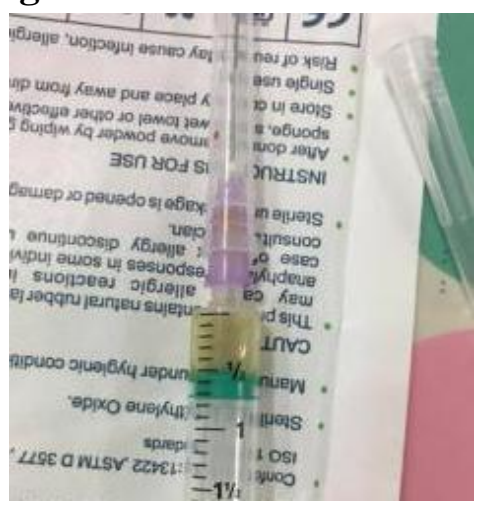

Figure 6: Serosanginous fluid aspirate from the skin and joint abscess.

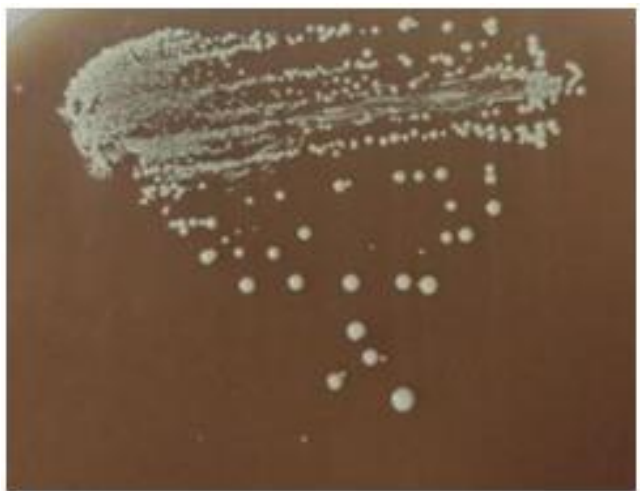

Figure 7: Chocolate agar demonstrating chalky white colonies.

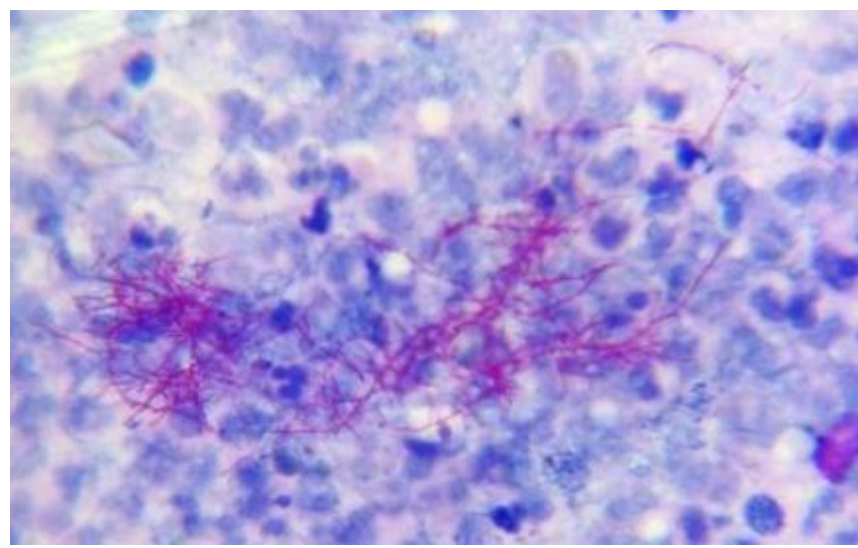

Figure 8: Modified Ziehl - Neelsen staining showing acid-fast bacilli which are filamentous, beaded, and branching bacteria

\section{Discussion}

Nocardiosis is a localized or distributed infection caused by gram-positive aerobic bacilli of the genus Nocardia, which has a positive effect on modified Ziehl-Neelsen staining and can occur in animals or humans. These bacilli are usually environmental saprophytes that live in soil, organic matter decomposing, or in water. The major species concerned are N.Asteroids (80 to 90 percent of cases), N. farcinica, N. nova, N. brasiliensis, N.otitidiscaviarum, and $\mathrm{N}$. transvalensis.

Disease transmission typically occurs through inhalation, with deposition in the lungs that can be spread to other locations. However, primary skin infection can result from direct inoculation of the micro-organism, such as that occurring during trauma or surgery. No evidence of human-tohuman or animal-to-human transmission exists.

Nocardiosis occurs in patients with defective cellular immunity. The clinical profile is nonspecific and must be suspected in patients with concomitant respiratory, cutaneous, or neurological manifestations. Therefore, it is included in the differential diagnosis of sarcoidosis, actinomycosis, and aspergillosis. The lung is the most frequently affected organ, and it must be noted that nocardia is not part of the natural flora of the respiratory tract, and its detection in respiratory fluid should therefore always be regarded as the possible causative agent 
of infection. The thoracic radiological appearance is often non-specific, including nodules or masses (isolated or multiple), with or without cavitation (with cavitation being more common), reticular infiltrates, alveolar consolidation, and pleural effusion.

For a conclusive diagnosis, it is important to isolate and classify the organism through the examination of the biopsy or aspirate from the infected sites or through the assessment of the respiratory fluid. It is important to notify the laboratory of suspected nocardiosis so that culture should be preserved for 20 or 30 days. Blood culture is rarely possible.

The antibiotic treatment prescribed depends on the severity and position of the infection and the immunity of the host. The trimethoprimsulfamethoxazole combination ( 5 to $10 \mathrm{mg} / \mathrm{kg} /$ day of TMP) is the recommended regimen for pleuropulmonary infections (with or without skin involvement) as well as for the primary cutaneous type. It is recommended that higher doses of trimethoprim-sulfamethoxazole $(15 \mathrm{mg} / \mathrm{kg} /$ day of TMP) or a combination of imipenem ( $2 \mathrm{~g} /$ day) and amikacin (10 to $15 \mathrm{mg} / \mathrm{kg} /$ day) be used for the first six weeks and that the trimethoprimsulfamethoxazole combination be maintained at lower doses (5 to $10 \mathrm{mg} / \mathrm{kg} /$ day of TMP) until the end of the treatment period. The length of treatment depends on the location of the lesions and on the host immunity. Treatment should be continued for three months for skin lesions compared to up to six months for pulmonary and systemic types (without CNS involvement). Treatment should be extended for 12 months in immunocompromised patients and in cases of CNS involvement. Clinical improvement occurs between seven and ten days. The combination of imipenem and amikacin can be used in patients that are intolerant or refractory to the trimethoprim-sulfamethoxazole combination, such as monotherapy with ceftriaxone, minocycline, linezolid, or a combination of amoxicillin and clavulanate. Antimicrobial susceptibility tests are recommended in refractory patients, patients with recurrence, and patients with hypersensitivity to the existing regimen.

Surgical care is recommended for the majority of patients with CNS lesions that are refractory to antibiotic therapy, with extensive skin involvement.

\section{Conclusion}

An increase in the number of patients undergoing immunosuppressive therapy for stem cell transplantation, hematological and solid tissue cancer, and autoinflammatory disorders ensure that Nocardia remains a formidable pathogen. Although this organism is capable of producing serious and metastatic disease in the appropriate host, early detection and initiation of appropriate treatment may lead to successful outcomes. Cure rates for patients with skin infection are average $>95 \%$. Ninety percent of pleuropulmonary infections can still be treated effectively.

\section{References}

1. Hino Y, Doki N, Senoo Y, Sekiya N, Kurosawa S, Tsuboi $S$, et al. Disseminated nocardiosis after unrelated bone marrow transplantation. Transplant Infectious Disease. 2016;18(6):942-945.

2. Jiang Y, Huang A, Fang Q. Disseminated nocardiosis caused by Nocardia otitidiscaviarum in an immunocompetent host: A case report and literature review. Experimental and therapeutic medicine. 2016;12(5):3339-3346.

3. Minero MV, Marín M, Cercenado E, Rabadán PM, Bouza E, Muñoz P. Nocardiosis at the turn of the century. Medicine. 2009;88(4):250-261.

4. Vijay Kumar GS, Mahale RP, Rajeshwari KG, Rajani R, Shankaregowda R. Primary facial cutaneous nocardiosis in an HIV patient and review of cutaneous nocardiosis in India. Indian J Sex Transm Dis AIDS. 2011;32(1):40-3.

5. Outhred AC, Watts MR, Chen SC, Sorrell TC. Nocardia infections of the face and 
neck. Current infectious disease reports. 2011;13(2):132-140.

6. Benzaquen M, Belenotti P, Lebowitz D, Drancourt M, Serratrice J. Primary cutaneous nocardiosis caused by Nocardia takedensis with pulmonary dissemination in an immunosuppressed patient. Australasian Journal of Dermatology. 2017;58(3):e97-e100.

7. B C, J T, Z L, N W, X G, F W. Primary Cutaneous Nocardiosis in a Patient With Nephrotic Syndrome: A Case Report and Review of the Literature [Internet]. Vol. 95, Medicine. Medicine (Baltimore); 2016 [cited 2020 Nov 16]. Available from: https://pubmed.ncbi.nlm.nih.gov/2681788 $5 /$ 